\title{
TRANSFORMS OF MEASURES ON QUOTIENTS AND SPLINE FUNCTIONS
}

\author{
BY \\ ALAN MACLEAN
}

\begin{abstract}
Let $G$ be a LCA group with closed subgroup $H$ and let $\nu \in M(G / H)$. A general procedure is established for constructing a large family of measures in $M(G)$ whose Fourier transforms interpolate $\hat{\nu}$. This method is used to extend a theorem of Shepp and Goldberg by showing that if $\nu \in M([0,2 \pi))$, then each even order cardinal spline function which interpolates the sequence $(\hat{v}(n))_{n--\infty}^{\infty}$ is the Fourier transform of a bounded Borel measure on $\boldsymbol{R}$.
\end{abstract}

The purpose of this article is to investigate certain relationships between the Fourier transforms of measures defined on the quotient $G / H$ of an LCA group $G$ and the Fourier transforms of measures defined on $G$ itself. Given a measure $\nu$ on $G / H$ we shall develop procedures by which the Fourier transform $\hat{\nu}$ may be extended so that it becomes the transform $\hat{\mu}$ of a measure on $G$. The existence of such extensions is, of course, well known [7, 2.7.2], [1, p. 99], viz., the Fourier transforms of measures on $G / H$ are exactly the restrictions of the Fourier transforms of measures on $G$. The emphasis here, however, is not on existence, but rather on the various ways that $\hat{\nu}$ may be extended. We shall develop a method by which a large family of extensions of $\hat{v}$ may be constructed in such a way that analytic properties of $\nu$ (absolute continuity, singularity, etc.) carry over to these extensions.

Our motivation stems from the following theorem, attributed to L. A. Shepp by Feller [3, p. 609], and proved independently and in detail by R. R. Goldberg [4] (an extension to $R^{n}$ is contained in [5]).

THEOREM [SHEPP-GoldBerG]. Let $\left(a_{n}\right)_{n=-\infty}^{\infty}$ be the sequence of Fourier-Stieltjes coefficients of a bounded Borel measure on $[0,2 \pi)$. Then the function whose graph consists of the line segments successively joining the points $\left(n, a_{n}\right)$ is the FourierStieltjes transform of a bounded Borel measure on $(-\infty, \infty)$.

Thus, the linear extension of the sequence $\left(a_{n}\right)_{n=-\infty}^{\infty}$ is the transform of a bounded measure on $R$. The question arises whether there is a class of higher degree polynomial extensions of $\left(a_{n}\right)_{n=-\infty}^{\infty}$ which are also transforms of bounded measures on $R$. Such extensions cannot behave too wildly off of the integers and this leads one to consider those extensions which satisfy certain smoothness

Received by the editors October 19, 1979 and, in revised form, December 5, 1979.

AMS (MOS) subject classifications (1970). Primary 43A05, 43A25, 42A72, 41A15.

Key words and phrases. LCA group, bounded Borel measure, Fourier-Stieltjes transform, spline function. 
conditions The polynomial extensions that we have in mind are the even order cardinal spline functions of Schoenberg [8], [11]. If $m$ is an even integer $>2$, then a cardinal spline function of order $m$ is, loosely speaking, a function obtained by joining together polynomials of degree $m-1$ at the integers in such a way that the resulting function is in $C^{m-2}(R)$ (definitions will be given later). It is known [10] that for each bounded sequence $\left(x_{n}\right)_{n=-\infty}^{\infty}$, and each even integer $m \geqslant 2$, that there is a unique bounded spline $S$ of order $m$ which interpolates $\left(x_{n}\right)_{n=-\infty}^{\infty}$, i.e., which has the property that $S(n)=x_{n}$ for each integer $n$. In particular, the unique bounded 2nd order spline which interpolates $\left(x_{n}\right)_{n=-\infty}^{\infty}$ is the linear extension of $\left(x_{n}\right)_{n=-\infty}^{\infty}$ to $R$. Thus, the theorem above states that the bounded 2 nd order spline which interpolates the Fourier-Stieltjes coefficients $\left(a_{n}\right)_{n=-\infty}^{\infty}$ is the Fourier-Stieltjes transform of a bounded measure on $R$. We shall extend the Shepp-Goldberg theorem by showing that for each even integer $m \geqslant 2$, the bounded $m$ th order spline function interpolating $\left(a_{n}\right)_{n--\infty}^{\infty}$ is also the Fourier-Stieltjes transform of a bounded measure $\omega_{m}$ on $R$.

The measures $\omega_{m}$ arise as special instances of a general construction applicable to all LCA groups, and we shall carry out the construction in this setting. In this sense, one might regard the transforms $\hat{\omega}$ of the measures to be constructed as generalized cardinal spline functions. As might be anticipated, the techniques of [4] are no longer available in the present context. It is perhaps of interest to compare the two situations briefly. Given a bounded Borel measure $\nu$ on $G / H$ we wish to produce a bounded Borel measure $\omega$ on $G$ which satisfies certain properties and for which $\hat{\omega}(\gamma)=\hat{\nu}(\gamma)$ for all $\gamma$ in the annihilator of $H$. In [4] one has $G=R$ and $H=2 \pi Z$, while the quotient $R / 2 \pi Z$ and the annihilator of $2 \pi Z$ are identified with $[0,2 \pi)$ and $Z$, respectively. The construction in [4] produces, for each measure $\nu$ on $[0,2 \pi)$, a measure $\omega$ on $R$ such that $\hat{\omega}$ is the linear extension to $R$ of $\hat{\nu}(n)=a_{n}$. The measure $\omega$ is defined to be the periodic extension of $\nu$ to $R$ multiplied by an appropriate weight function, and the proof that $\hat{\omega}$ is indeed the linear extension of $\hat{\nu}$ is based on the fact that the $(C, 1)$ means of the Fourier series of $\nu$ converge weak-* to $\nu$. In our more general setting we lose both the compactness of the quotient $G / H$ and the use of $(C, 1)$ summability. The lack of compactness is compensated for by initially restricting attention to measures $\nu$ with compact support in $G / H$. The supports are used to produce auxiliary weight functions for which the Poisson summation formula holds, and these, in turn, give rise to measures $\omega$ whose transforms are the analogues of the linear extensions above. Thus, the arguments involving $(C, 1)$ summability are circumvented, in effect, by appealing to the Poisson summation formula.

The author wishes to thank Professor A. Elcrat for valuable conversations regarding spline functions.

Throughout $G$ will denote a locally compact abelian (LCA) group with a fixed Haar measure $d x$. We denote the dual group of $G$ by $\Gamma$. For a closed subgroup $H$ of $G$ we let $\pi: G \rightarrow G / H$ be the natural quotient map and denote the elements of $G / H$ by $\dot{x}$. Thus, $\pi(x)=\dot{x}$. We identify the dual of $G / H$, as usual, with the annihilator $H^{\perp}=\{\gamma \in \Gamma: \gamma(h)=1, h \in H\}$ of $H$. To emphasize that $H^{\perp}$ is 
being regarded as the dual of $G / H$ we shall, unless otherwise specified, denote the elements of $H^{\perp}$ by $\gamma$ and the elements of $\Gamma$ by $\chi$. In particular, $d \gamma$ and $d \chi$ denote Haar measures on $H^{\perp}$ and $\Gamma$ respectively. $C(G)$ is the space of bounded continuous complex functions on $G . C_{0}(G)$ and $C_{00}(G)$ are the subspaces of $C(G)$ consisting of functions which vanish at infinity and functions with compact support respectively. $M(G)$ is the space of all bounded complex Borel measures on $G$.

Let $H$ be a closed subgroup of $G$ with fixed Haar measure $d h$. The linear mapping $T: C_{00}(G) \rightarrow C_{00}(G / H)$ defined by

$$
T f(\dot{x})=\int_{H} f(x+h) d h
$$

maps $C_{00}(G)$ onto $C_{00}(G / H)$, and there is a Haar measure $d \dot{x}$ on $G / H$ such that the "Weil formula"

$$
\int_{G} f(x) d x=\int_{G / H} T f(\dot{x}) d \dot{x}=\int_{G / H} \int_{H} f(x+h) d h d \dot{x}
$$

holds for all $f \in C_{00}(G)$. We assume throughout that $d \dot{x}$ has been chosen in this manner. The map $T$ extends to a norm decreasing algebra homomorphism of $L^{1}(G)$ onto $L^{1}(G / H)$ such that (2) holds, where now $T f$ is defined almost everywhere on $G / H$ by (1) [6, p. 74].

The Fourier(-Stieltjes) transform of $\mu \in M(G)$ is defined on $\Gamma$ by

$$
\hat{\mu}(\chi)=\int_{G} \overline{\chi(x)} d \mu(x) .
$$

We identify $f \in L^{1}(G)$ with the absolutely continuous measure $f(x) d x \in M(G)$. The Fourier transform of $f$ is then $\hat{f}(\chi)=\int_{G} f(x) \overline{\chi(x)} d x$. The inverse Fourier transform $\check{f}$ of $f \in L^{1}(G)$ is defined by $\check{f}(\chi)=\hat{f}(-\chi)$. It will be convenient to regard the Fourier algebra $A(G)$ to be defined as $A(G)=\left\{\check{g}: g \in L^{1}(\Gamma)\right\}$.

In the construction to follow we shall utilize certain auxiliary functions. The existence of such functions is established in the following lemma.

Lemma. Let $H$ be a closed subgroup of $G$ and let $K$ be a compact subset of $G / H$. Then there exist continuous functions $\delta$ on $G$ such that $\delta \in L_{+}^{1}(G), \hat{\delta} \in C_{00}^{+}(\Gamma)$, and $T \delta$ is continuous on $G / H$ with $T \delta(\dot{x})>0$ for all $\dot{x} \in K$.

Proof. We note first that if $C$ is any compact subset of $\Gamma$, then there exists an $f \in L_{+}^{1}(G) \cap C_{0}(G)$ such that $\hat{f}>0$ on $C$ and $\hat{f} \in C_{00}^{+}(\Gamma)$. For we may choose an $h \in L^{1}(G) \cap L^{2}(G)$ such that $\hat{h}=1$ on $C \cup\{0\}$ and $\hat{h} \in C_{00}^{+}(\Gamma)$. We may assume that $h \in C_{0}(G)$ by the inversion theorem. Then $f=|h|^{2}$ is easily seen to satisfy the required conditions.

Now, let $F$ be a compact subset of $G$ such that $\pi(F)=K$. By the previous paragraph there is an $f \in L_{+}^{1}(G) \cap C_{0}(G)$ such that $\hat{f}(0)>0$ and $\hat{f} \in C_{00}^{+}(\Gamma)$, and there is a $g \in L_{+}^{1}(\Gamma) \cap C_{0}(\Gamma)$ such that $\check{g}>0$ on $F$ and $\check{g} \in C_{00}^{+}(G)$. Then $f * \check{g} \in L_{+}^{1}(G) \cap C_{0}(G)$ and $(f * \check{g})^{\wedge}=\hat{f} g \in C_{00}^{+}(\Gamma)$. For each $x \in G$, we have

$$
\int_{H} f * \check{g}(x+h) d h \leqslant\|f\|_{t}\|T \mid \check{g}\|_{\infty} \text {. }
$$


Thus, $T(f * \check{g})$ is defined everywhere on $G / H$ and it follows that $T(f * \check{g})=$ $T f * T \check{g}$ everywhere on $G / H$. Since $T \check{g} \in C_{00}(G / H), T(f * \check{g})$ is continuous on $G / H$. If $x \in F$, then since $\hat{f}(0)>0$ we have $f(0) \check{g}(x)=\check{g}(x) \int_{\Gamma} \hat{f}(\chi) d \chi>0$. Hence, $f * \check{g}(x)=\int_{G} f(y) \check{g}(x-y) d y>0$ for all $x \in F$. Then $T(f * \check{g})(\dot{x})=$ $\int_{H} f * \check{g}(x+h) d h>0$ for all $\dot{x} \in K$.

Let $\nu$ be a fixed measure in $M(G / H)$. We wish to construct a certain family of measures $\omega \in M(G)$ with the property that the Fourier transform of each such $\omega$ interpolates $\hat{\nu}$, i.e., such that $\hat{\omega}(\gamma)=\hat{\nu}(\gamma)$ for all $\gamma \in H^{\perp}$. We proceed as follows.

Use the regularity of $\nu$ to select inductively disjoint compact subsets $K_{1}, K_{2}, \ldots$ of $G / H$ such that

$$
|\nu|\left(\left[\bigcup_{1}^{n} K_{j}\right]^{c}\right)<1 / n
$$

for all $n \geqslant 1$. Let $\nu_{n}$ be the measure in $M(G / H)$ obtained by restricting $\nu$ to $K_{n}$. Then since the $K_{n}$ 's are disjoint we have by (3) that

$$
\|\nu\|=\sum_{1}^{\infty}\left\|\nu_{n}\right\|
$$

(cf. [1, p. 99]).

For each $n \geqslant 1$, let $\delta_{n}$ be a continuous function on $G$ such that

$$
\begin{aligned}
& \delta_{n} \in L_{+}^{1}(G), \hat{\delta}_{n} \in C_{00}(\Gamma) \text { and } T \delta_{n} \text { is continuous } \\
& \text { on } G / H \text { with } T \delta_{n}>0 \text { on } K_{n} .
\end{aligned}
$$

Such functions exist by the lemma. We have, in fact, that $T \delta_{n} \in A^{+}(G / H)$. For $\hat{\delta}_{n} \mid H^{\perp} \in L^{1}\left(H^{\perp}\right)$ and since, as is well known, $\left(T \delta_{n}\right)^{\wedge}=\hat{\delta}_{n} \mid H^{\perp}$ it follows from the inversion theorem and the continuity of $T \delta_{n}$ that $T \delta_{n}=\left(\hat{\delta}_{n} \mid H^{\perp}\right)^{-}$. Then since $T \delta_{n}>0$ on the compact set $K_{n}$ we may apply the Wiener-Levy theorem to obtain a function $\check{\beta}_{n} \in A(G / H)$ such that

$$
\check{\beta}_{n} \cdot T \delta_{n}=1 \text { on } K_{n} \text {. }
$$

Since $T \delta_{n} \geqslant 0$ on $G / H$ we may assume that $\check{\beta}_{n} \geqslant 0$. Finally, define $\psi_{n}: G \rightarrow C$ by

$$
\psi_{n}=\delta_{n} \cdot\left(\check{\beta}_{n} \circ \pi\right) \text {. }
$$

Theorem 1. Let $\nu \in M(G / H)$ and let $\nu_{n}$ and $\psi_{n}$ be as defined above. Then there exists a measure $\omega \in M(G)$ such that

(i) $\hat{\omega} \mid H^{\perp}=\hat{\nu}$ and $\hat{\omega}(\chi)=\Sigma_{1}^{\infty} \int_{H^{\perp}} \hat{\nu}_{n}(\gamma) \hat{\psi}_{n}(\chi-\gamma) d \gamma$ for all $\chi \in \Gamma$.

(ii) If $f \in L^{1}(|\nu|)$, then $f \circ \pi \in L^{1}(|\omega|)$ and $\int_{G} f \circ \pi d \omega=\int_{G / H} f d \nu$ and $\int_{G} f \circ \pi d|\omega|=\int_{G / H} f d|\nu|$.

(iii) For each Borel set $E \subseteq G / H, \nu(E)=\omega\left(\pi^{-1}(E)\right)$ and $|\nu|(E)=|\omega|\left(\pi^{-1}(E)\right)$. In particular, $\|\nu\|=\|\omega\|$.

(iv) Let $1 \leqslant p \leqslant \infty$. If $f \in L^{p}(|\nu|)$, then $f \circ \pi \in L^{p}(|\omega|)$ and $\|f\|_{L^{p}(|\nu|)}=$ $\|f \circ \pi\|_{L^{p}(|\boldsymbol{|}|)}$. 
Proof. Since $\delta_{n}$ is continuous and in $L_{+}^{1}(G)$ and $\check{\beta}_{n} \circ \pi \in C_{+}(G)$ we have that $\psi_{n}$ is continuous and in $L_{+}^{1}(G)$. We have

$$
\begin{aligned}
\hat{\psi}_{n}(\chi) & =\int_{G} \delta_{n}(x) \check{\beta}_{n}(\dot{x}) \overline{\chi(x)} d x=\int_{H^{\perp}} \int_{G} \beta_{n}(\gamma) \delta_{n}(x) \overline{(\chi-\gamma)(x)} d x d \gamma \\
& =\int_{H^{\perp}} \beta_{n}(\gamma) \hat{\delta}_{n}(\chi-\gamma) d \gamma .
\end{aligned}
$$

We assert that the extended "Poisson Formula" holds for $\psi_{n}$, i.e., that

$$
\int_{H} \psi_{n}(x+h) \overline{\chi(x+h)} d h=\int_{H^{\perp}} \hat{\psi}_{n}(\chi+\gamma) \gamma(\dot{x}) d \gamma
$$

for all $x \in G, \chi \in \Gamma$. This is seen as follows. First, (9) holds for $\delta_{n}$ since $\delta_{n} \in L^{1}(G)$ is continuous and $\hat{\delta}_{n} \in C_{00}(\Gamma)\left[6\right.$, p. 122]. Fix $\chi \in \Gamma$. Then $T\left(\bar{\chi} \psi_{n}\right) \in$ $L^{1}(G / H)$ and, moreover, $T\left(\bar{\chi} \psi_{n}\right)$ is continuous on $G / H$;

$$
\begin{aligned}
T\left(\bar{\chi} \psi_{n}\right)(\dot{x}) & =\int_{H} \psi_{n}(x+h) \overline{\chi(x+h)} d h=\check{\beta}_{n}(\dot{x}) \int_{H} \delta_{n}(x+h) \overline{\chi(x+h)} d h \\
& =\check{\beta}_{n}(\dot{x}) \int_{H^{\perp}} \hat{\delta}_{n}(\chi+\gamma) \gamma(\dot{x}) d \gamma
\end{aligned}
$$

where $\check{\beta}_{n}$ and $\dot{x} \rightarrow \int_{H^{\perp}} \hat{\delta}_{n}(\chi+\gamma) \gamma(\dot{x}) d \gamma$ are continuous on $G / H$. Further, $T\left(\bar{\chi} \psi_{n}\right)^{\wedge} \in L^{1}\left(H^{\perp}\right)$ since by $(8)$

$$
\begin{aligned}
\int_{H^{\perp}}\left|T\left(\bar{\chi} \psi_{n}\right)^{\wedge}(\gamma)\right| d \gamma & =\int_{H^{\perp}}\left|\hat{\psi}_{n}(\chi+\gamma)\right| d \gamma \leqslant \int_{H^{\perp}} \int_{H^{\perp}}\left|\beta_{n}(\sigma) \hat{\delta}_{n}(\chi+\gamma-\sigma)\right| d \sigma d \gamma \\
& =\left\|\beta_{n}\right\|_{L^{\perp}\left(H^{\perp}\right)} \int_{H^{\perp}}\left|\hat{\delta}_{n}(x+\gamma)\right| d \gamma<\infty .
\end{aligned}
$$

Thus, by inversion $T\left(\bar{\chi} \psi_{n}\right)(\dot{x})=\left[T\left(\bar{\chi} \psi_{n}\right)^{\wedge}\right]^{\sim}(\dot{x})$ for all $\dot{x} \in G / H$, which is formula (9). We note further that by setting $\chi=0$ in (10) we obtain

$$
T \psi_{n}(\dot{x})=\beta_{n}(\dot{x}) \int_{H} \delta_{n}(x+h) d h=\check{\beta}_{n}(\dot{x}) T \delta_{n}(\dot{x}) .
$$

In particular, it follows from (6) that

$$
T \psi_{n}=1 \text { on } K_{n} .
$$

Now, define $\omega_{n}$ on $G$ by setting

$$
\int_{G} f d \omega_{n}=\int_{G / H} T\left(f \psi_{n}\right) d \nu_{n}=\int_{G / H} \int_{H} f(x+h) \psi_{n}(x+h) d h d \nu_{n}
$$

for all $f \in C_{00}(G)$. Then by (12)

$$
\left|\int_{G} f d \omega_{n}\right| \leqslant \int_{G / H}\left|T\left(f \psi_{n}\right)\right| d\left|\nu_{n}\right|<\|f\|_{\infty} \int_{G / H} T \psi_{n}(\dot{x}) d\left|\nu_{n}\right|=\|f\|_{\infty}\left\|\nu_{n}\right\|
$$

for each $f \in C_{00}(G)$, and it follows that $\omega_{n} \in M(G)$ with $\left\|\omega_{n}\right\|<\left\|\nu_{n}\right\|$. If $f$ is nonnegative and lower semicontinuous on $G$, then by a standard argument (cf. [6, p. 60]) $T\left(f \psi_{n}\right)$ is nonnegative and lower semicontinuous on $G / H$ and

$$
\int_{G} f d\left|\omega_{n}\right| \leqslant \int_{G / H} \int_{H} f(x+h) \psi_{n}(x+h) d h d\left|\nu_{n}\right|
$$


Similarly, by decomposing $\nu_{n}$ into a sum of positive measures and first considering nonnegative lower semicontinuous functions one sees that (13) holds for bounded continuous functions on $G$. In particular, for $\gamma \in H^{\perp}$ we obtain again using (12) that

$$
\begin{aligned}
\hat{\omega}_{n}(\gamma) & =\int_{G / H} \int_{H} \overline{\gamma(x+h)} \psi_{n}(x+h) d h d \nu_{n} \\
& =\int_{G / H} \overline{\gamma(\dot{x})} T \psi_{n}(\dot{x}) d \nu_{n}=\hat{\nu}_{n}(\gamma) .
\end{aligned}
$$

Hence, $\hat{\omega}_{n} \mid H^{\perp}=\hat{\nu}_{n}$. For general $\chi \in \Gamma$ we apply (9) to obtain

$$
\begin{aligned}
\hat{\omega}_{n}(\chi) & =\int_{G / H} \int_{H} \overline{\chi(x+h)} \psi_{n}(x+h) d h d \nu_{n}=\int_{G / H} \int_{H^{\perp}} \overline{\gamma(\dot{x})} \hat{\psi}_{n}(\chi-\gamma) d \gamma d \nu_{n} \\
& =\int_{H^{\perp}} \int_{G / H} \overline{\gamma(\dot{x})} \hat{\psi}_{n}(\chi-\gamma) d \nu_{n} d \gamma=\int_{H^{\perp}} \hat{\nu}_{n}(\gamma) \hat{\psi}_{n}(\chi-\gamma) d \gamma
\end{aligned}
$$

where the use of Fubini's theorem is justified by (11).

We have $\left\|\omega_{n}\right\| \leqslant\left\|\nu_{n}\right\|$ for all $n \geqslant 1$ and since, by (4), $\|\nu\|=\Sigma_{1}^{\infty}\left\|\nu_{n}\right\|$ it follows that $\left(\sum_{1}^{n} \omega_{j}\right)_{n=1}^{\infty}$ is Cauchy in $M(G)$. Thus, there is a measure $\omega \in M(G)$ such that $\omega=\Sigma_{1}^{\infty} \omega_{n}$. If $\gamma \in H^{\perp}$, then it follows from (3) and (15) that

$$
\hat{\omega}(\gamma)=\sum_{1}^{\infty} \hat{\omega}_{n}(\gamma)=\sum_{1}^{\infty} \hat{\nu}_{n}(\gamma)=\hat{\nu}(\gamma)
$$

Hence, $\hat{\omega} \mid H^{\perp}=\hat{\nu}$. If $\chi \in \Gamma$, then (16) shows that

$$
\hat{\omega}(\chi)=\sum_{1}^{\infty} \int_{H^{\perp}} \hat{\nu}_{n}(\gamma) \hat{\psi}_{n}(\chi-\gamma) d \gamma .
$$

Thus, (i) holds.

To establish (ii) we argue as follows. In view of (15) we have that

$$
\int_{G} p \circ \pi d \omega_{n}=\int_{G / H} p d v_{n}
$$

for each trigonometric polynomial $p$ on $G / H$ and $n \geqslant 1$. Let $f \in L^{1}(|v|)$. Fix $n$ and choose trigonometric polynomials $p_{m}$ such that $\left\|p_{m}-f\right\|_{L^{\prime}\left(p_{n}\right)} \rightarrow 0$. By passing to a subsequence we may suppose that $p_{m}(\dot{x}) \rightarrow f(\dot{x})$ for all $\dot{x} \notin E$ where $E$ is a Borel subset of $G / H$ such that $\left|\nu_{n}\right|(E)=0$. We have from (12) and (14) that

$$
\begin{aligned}
\left\|p_{m} \circ \pi-p_{k} \circ \pi\right\|_{L^{\prime}\left(\left|\omega_{n}\right|\right)} & \leqslant \int_{G / H} \int_{H}\left|p_{m} \circ \pi-p_{k} \circ \pi\right|(x+h) \psi_{n}(x+h) d h d\left|\nu_{n}\right| \\
& =\int_{G / H}\left|p_{m}(\dot{x})-p_{k}(\dot{x})\right| T \psi_{n}(\dot{x}) d\left|\nu_{n}\right|=\left\|p_{m}-p_{k}\right\|_{L^{\prime}\left(\left|p_{n}\right|\right)}
\end{aligned}
$$

Thus, $\left(p_{m} \circ \pi\right)_{m=1}^{\infty}$ is Cauchy in $L^{1}\left(\left|\omega_{n}\right|\right)$. Let $p_{m} \circ \pi \rightarrow g$ in $L^{1}\left(\left|\omega_{n}\right|\right)$. Again by passing to a subsequence we may assume that $p_{m} \circ \pi(x) \rightarrow g(x)$ for all $x \notin A$ where $A$ is a Borel subset of $G$ such that $\left|\omega_{n}(A)\right|=0$. Suppose for the moment that $\left|\omega_{n}\right|\left(\pi^{-1}(E)\right)=0$. Then for each $x \notin A \cup \pi^{-1}(E)$ we have

$$
g(x)=\lim _{m \rightarrow \infty} p_{m} \circ \pi(x)=\lim _{m \rightarrow \infty} p_{m}(\dot{x})=f(\dot{x})=f \circ \pi(x) .
$$


Hence, $g=f \circ \pi,\left|\omega_{n}\right|$-a.e. Consequently, $f \circ \pi \in L^{1}\left(\left|\omega_{n}\right|\right)$ and by (17) we have

$$
\int_{G} f \circ \pi d \omega_{n}=\lim _{m \rightarrow \infty} \int_{G} p_{m} \circ \pi d \omega_{n}=\lim _{m \rightarrow \infty} \int_{G / H} p_{m} d \nu_{n}=\int_{G / H} f d \nu_{n} .
$$

We also have that $\left\|\left|p_{m}\right|-|f|\right\|_{L^{\prime}\left(\left|\nu_{n}\right|\right)} \rightarrow 0$ and $\left\|\left|p_{m}\right| \circ \pi-|f| \circ \pi\right\|_{L^{\prime}\left(\left|\omega_{n}\right|\right)} \rightarrow 0$. It follows from (12) and (14) once again that

$$
\int_{G}|f| \circ \pi d\left|\omega_{n}\right|=\lim _{m \rightarrow \infty} \int_{G}\left|p_{m}\right| \circ \pi d\left|\omega_{n}\right| \leqslant \lim _{m \rightarrow \infty} \int_{G / H}\left|p_{m}\right| d\left|\nu_{n}\right|=\int_{G / H}|f| d\left|\nu_{n}\right| .
$$

Thus, we have shown that if $f \in L^{1}(|\nu|)$, then $f \circ \pi \in L^{1}\left(\left|\omega_{n}\right|\right)$ for all $n>1$ and

$$
\sum_{1}^{\infty} \int_{G}|f| \circ \pi d\left|\omega_{n}\right| \leqslant \sum_{1}^{\infty} \int_{G / H}|f| d\left|\nu_{n}\right|=\int_{G / H}|f| d|\nu|<\infty
$$

Since $\omega=\Sigma_{1}^{\infty} \omega_{n}$ in $M(G)$ it now follows from the dominated convergence theorem that $f \circ \pi \in L^{1}(|\omega|)$ and

$$
\int_{G} f \circ \pi d \omega=\sum_{1}^{\infty} \int_{G} f \circ \pi d \omega_{n}=\sum_{1}^{\infty} \int_{G / H} f d \nu_{n}=\int_{G / H} f d \nu .
$$

Thus, the first part of (ii) will be established provided we verify that $\left|\omega_{n}\right|\left(\pi^{-1}(E)\right)$ $=0$ if $\left|\nu_{n}\right|(E)=0$. Let $\varepsilon>0$ and choose an open set $V \subseteq G / H$ such that $\left|\nu_{n}\right|(V)<\varepsilon$. Then since $\xi_{V} \circ \pi$ is nonnegative and lower semicontinuous on $G$ we have

$$
\begin{aligned}
\left|\omega_{n}\right|\left(\pi^{-1}(E)\right) & \leqslant \int_{G} \xi_{\pi^{-1}(V)}(x) d\left|\omega_{n}\right|<\int_{G} \xi_{V} \circ \pi(x) d\left|\omega_{n}\right| \\
& \leqslant \int_{G / H} \xi_{V}(\dot{x}) T \psi_{n}(\dot{x}) d\left|\nu_{n}\right|=\left|\nu_{n}\right|(V)<\varepsilon .
\end{aligned}
$$

Hence, $\left|\omega_{n}\right|\left(\pi^{-1}(E)\right)=0$.

Now, if $E$ is a Borel set in $G / H$, then (19) shows that $\nu(E)=\omega\left(\pi^{-1}(E)\right)$. Then $\sum_{1}^{n}\left|\nu\left(E_{j}\right)\right|=\sum_{1}^{n}\left|\omega\left(\pi^{-1}\left(E_{j}\right)\right)\right|$, if $E=\bigcup_{1}^{n} E_{j}$ and $E_{j} \cap E_{k}=\varnothing$, and so

$$
\begin{aligned}
|\nu|(E) & =\sup \left\{\sum_{1}^{n}\left|\nu\left(E_{j}\right)\right|: E=\bigcup_{1}^{n} E_{j}, E_{j} \cap E_{k}=\varnothing\right\} \\
& \leqslant \sup \left\{\sum_{1}^{m}\left|\omega\left(B_{j}\right)\right|: \pi^{-1}(E)=\bigcup_{1}^{m} B_{j}, B_{j} \cap B_{k}=\varnothing\right\}=|\omega|\left(\pi^{-1}(E)\right) .
\end{aligned}
$$

But by (18)

$$
|\omega|\left(\pi^{-1}(E)\right)=\int_{G} \xi_{E} \circ \pi d|\omega| \leqslant \sum_{1}^{\infty} \int_{G} \xi_{E} \circ \pi d\left|\omega_{n}\right| \leqslant \int_{G / H} \xi_{E} d|\nu|=|\nu|(E) .
$$

Thus, in fact, $|\nu|(E)=|\omega|\left(\pi^{-1}(E)\right)$ for all Borel sets $E \subset G / H$. In particular, $\|\nu\|=\|\omega\|$. Approximation by simple functions now shows that $\int_{G} f \circ \pi d|\omega|=$ $\int_{G / H} f d|\nu|$ for all $f \in L^{1}(|\nu|)$. Thus, (ii) and (iii) hold and the remaining assertions follow easily. 
TheOREM 2. Let $\nu$ and $\omega$ be as in Theorem 1.

(i) If $\nu$ is absolutely continuous with Radon-Nikodym derivative $f \in L^{1}(G / H)$, then $\omega$ is absolutely continuous and the Radon-Nikodym derivative of $\omega$ is locally almost everywhere equal to $\Sigma_{1}^{\infty} \psi_{n}\left[\left(f \xi_{K_{n}}\right) \circ \pi\right]$.

(ii) If $\nu$ is continuous, then $\omega$ is continuous.

(iii) If $\nu$ is singular, then $\omega$ is singular.

Proof. If $\nu$ is continuous, then the continuity of $\omega$ follows from Theorem 1(iii).

Next assume that $\nu$ is singular. To show that $\omega$ is singular we apply a theorem of Doss [2, Theorem 1]. Let $\varepsilon>0$ and let $K$ be compact in $\Gamma$. Then $K \cap H^{\perp}$ is compact in $H^{\perp}$ and since $\nu$ is singular there is by [2] a trigonometric polynomial $p=\Sigma_{1}^{n} c_{j} \gamma_{j}$ on $G / H$ with $\gamma_{j} \in H^{\perp} \cap K^{c}$ and $\|p\|_{\infty}<1$ such that $\left|\Sigma_{1}^{n} c_{j} \hat{\nu}\left(\gamma_{j}\right)\right|>$ $\|\nu\|-\varepsilon$. In view of our identification of $H^{\perp}$ and the dual of $G / H$ we may regard $p$ as a trigonometric polynomial on $G$. Then still $\|p\|_{\infty}<1$ and $\gamma_{j} \in K^{c}$. Since $\left|\sum_{1}^{n} c_{j} \hat{\omega}\left(\gamma_{j}\right)\right|=\left|\sum_{1}^{n} c_{j} \hat{\nu}\left(\gamma_{j}\right)\right|>\|\nu\|-\varepsilon=\|\omega\|-\varepsilon$ by Theorem 1, a second application of [2] shows that $\omega$ is singular.

Finally, assume that $\nu$ is absolutely continuous, say $\nu=f(\dot{x}) d \dot{x}$ where $f \in$ $L^{1}(G / H)$. Then $\nu_{n}=f \xi_{K_{n}} d \dot{x}$; notation as in Theorem 1. Choose $g_{m} \in C_{00}(G / H)$ such that $\left\|g_{m}-f\right\|_{L^{\prime}(G / H)} \rightarrow 0$. We may suppose that $g_{m}(\dot{x}) \rightarrow f(\dot{x})$ for all $\dot{x} \notin E$ where $E$ is a Borel set in $G / H$ such that $m_{G / H}(E)=0\left(m_{G / H}=d \dot{x}\right)$. We have

$$
\left\|\psi_{n}\left[\left(g_{m} \xi_{k_{n}}\right) \circ \pi\right]-\psi_{n}\left[\left(g_{l} \xi_{K_{n}}\right) \circ \pi\right]\right\|_{L^{\prime}(G)}<\left\|g_{m}-g_{l}\right\|_{L^{1}(G / H)}
$$

by (12) so that $\left(\psi_{n}\left[\left(g_{m} \xi_{K_{n}}\right) \circ \pi\right]\right)_{m=1}^{\infty}$ is Cauchy in $L^{1}(G)$. Let $\psi_{n}\left[\left(g_{m} \xi_{K_{n}}\right) \circ \pi\right] \rightarrow F$ in $L^{1}(G)$. We may suppose that $\psi_{n}\left[\left(g_{m} \xi_{K_{n}}\right) \circ \pi\right](x) \rightarrow F(x)$ for all $x \notin A$ where $A$ is a Borel set in $G$ with $m_{G}(A)=0$. For $x \notin A \cup \pi^{-1}(E)$ we have

$$
\begin{aligned}
F(x) & =\lim _{m \rightarrow \infty} \psi_{n}(x) \cdot\left(g_{m} \xi_{K_{n}} \circ \pi\right)(x) \\
& =\lim _{m \rightarrow \infty} \psi_{n}(x) g_{m}(\dot{x}) \xi_{K_{n}}(\dot{x})=\psi_{n}(x)\left(f \xi_{K_{n}}\right) \circ \pi(x) .
\end{aligned}
$$

Now, $\pi^{-1}(E)$ is locally null in $G\left[6\right.$, p. 66] so $F=\psi_{n}\left[\left(f \xi_{K_{n}}\right) \circ \pi\right]$ 1.a.e. on $G$. We have for $\chi \in \Gamma$

$$
\begin{aligned}
\hat{F}(\chi) & =\lim _{m \rightarrow \infty} \int_{G} \psi_{n}(x) \cdot\left(g_{m} \xi_{K_{n}}\right) \circ \pi(x) \overline{\chi(x)} d x \\
& =\lim _{m \rightarrow \infty} \int_{G / H} T\left[\psi \cdot\left(g_{m} \xi_{K_{n}}\right) \circ \pi \cdot \bar{\chi}\right](\dot{x}) d \dot{x} \\
& =\lim _{m \rightarrow \infty} \int_{G / H}\left[\int_{H} \hat{\psi}_{n}(\chi-\gamma) \overline{\gamma(\dot{x})} d \gamma\right] g_{m}(\dot{x}) \xi_{K_{n}}(\dot{x}) d \dot{x} \\
& =\int_{G / H} \int_{H^{\perp}} \hat{\psi}_{n}(\chi-\gamma) \overline{\gamma(\dot{x})} f(\dot{x}) \xi_{K_{n}}(\dot{x}) d \gamma d \dot{x} \\
& =\int_{H^{\perp}} \hat{\psi}_{n}(\chi-\gamma)\left(f \xi_{K_{n}}\right)^{\wedge}(\gamma) d \gamma=\int_{H^{\perp}} \hat{\nu}_{n}(\gamma) \hat{\psi}_{n}(x-\gamma) d \gamma=\hat{\omega}_{n}(x)
\end{aligned}
$$

where we have used the fact that $\left[T\left(\bar{\chi} \psi_{n}\right)^{\wedge}\right]^{\mu}(\dot{x})=\int_{H^{\perp}} \hat{\psi}_{n}(\chi-\gamma) \overline{\gamma(\dot{x})} d \gamma$ is bounded and continuous on $G / H$. It follows that $\omega_{n}$ is absolutely continuous. Then $\omega=\Sigma_{1}^{\infty} \omega_{n}$ is also. The Radon-Nikodym derivative of $\omega_{n}$ equals $\psi_{n}\left[\left(f \xi_{K_{n}}\right) \circ \pi\right]$ l.a.e. 
and it follows that the Radon-Nikodym derivative of $\omega$ is locally almost everywhere equal to $\Sigma_{1}^{\infty} \psi_{n}\left[\left(f \xi_{K_{n}}\right) \circ \pi\right]$ and the proof is complete.

Thus, if $\nu \in M(G / H)$, then each sequence $\left\{K_{n}\right\}$ of compact subsets of $G / H$ which satisfies (3), each sequence of functions $\left(\delta_{n}\right)$ which satisfies (5), and each sequence $\left(\check{\beta}_{n}\right)$ satisfying (6), determines a measure $\omega \in M(G)$ such that $\hat{\omega}$ is an extension of $\hat{\nu}: H^{\perp} \rightarrow C$ to $\Gamma$. A special case which is of interest in what follows occurs when $K$ is a fixed compact subset of $G / H$. Let $M(K)$ denote the subspace of $M(G / H)$ consisting of all $\nu \in M(G / H)$ such that supp $|\nu| \subseteq K$. Choose $\delta \in$ $L_{+}^{1}(G)$ and $\check{\beta} \in A^{+}(G / H)$ which satisfy (5) and (6) with $K_{n}$ replaced by $K$. Let $\psi=\delta \cdot(\check{\beta} \circ \pi)$ as before. We may then apply the above construction to each $\nu \in M(K)$ with $\psi$ fixed to obtain a measure $\nu_{\psi} \in M(G)$ which possesses the properties described in Theorems 1 and 2. In particular, for each $\nu \in M(K)$ we have $\hat{\nu}_{\psi} \mid H^{\perp}=\hat{\nu}$ and $\hat{\nu}_{\psi}(\chi)=\int_{H^{\perp}} \hat{\nu}(\gamma) \hat{\psi}(\chi-\gamma) d \gamma$ for all $\chi \in \Gamma$, and it follows easily that the map $\Lambda: M(K) \rightarrow M(G)$ defined by $\Lambda(\nu)=\nu_{\psi}$ is linear. It is also an isometry by Theorem 1(iii). These considerations apply to each $\nu \in M(G / H)$ in case $G / H$ itself is compact. We record this special case as a corollary.

Corollary. Assume that $G / H$ is compact. Let $\delta \in L_{+}^{1}(G) \cap C(G)$ be such that $\hat{\delta} \in C_{00}(\Gamma), T \delta \in C(G / H)$ and $T \delta>0$ on $G / H$. Let $\check{\beta} \in A^{+}(G / H)$ satisfy $\check{\beta} \cdot T \delta=1$ on $G / H$. Define $\psi$ on $G$ by $\psi=\delta \cdot(\check{\beta} \circ \pi)$. Then for each $\nu \in M(G / H)$ there exists a measure $\nu_{\psi} \in M(G)$ such that

(i) $\hat{\nu}_{\psi} \mid H^{\perp}=\hat{\nu}$ and $\hat{\nu}_{\psi}(\chi)=\Sigma_{\gamma \in H^{\perp}} \hat{\nu}(\gamma) \hat{\psi}(\chi-\gamma)$ for all $\chi \in \Gamma$.

(ii) If $f \in L^{1}(|\nu|)$, then $f \circ \pi \in L^{1}\left(\left|\nu_{\psi}\right|\right), \int_{G} f \circ \pi d \nu_{\psi}=\int_{G / H} f d \nu$, and $\int_{G} f \circ \pi d\left|\nu_{\psi}\right|=\int_{G / H} f d|\nu|$.

(iii) For each Borel set $E \subseteq G / H, \nu(E)=\nu_{\psi}\left(\pi^{-1}(E)\right)$ and $|\nu|(E)=\left|\nu_{\psi}\right|\left(\pi^{-1}(E)\right)$. In particular, $\|\nu\|=\left\|\nu_{\psi}\right\|$.

The map $\nu \rightarrow \nu_{\psi}$ is a linear isometry of $M(G / H)$ into $M(G)$.

As an application of the foregoing, and in particular of the corollary, we next prove the extension of the Shepp-Goldberg Theorem to spline functions discussed earlier.

A function $S$ defined on $R$ is called a cardinal spline function of degree $m-1$, where $m$ is an even natural number $\geqslant 2$, if $S \in C^{m-2}(R)$ and $S$ is a polynomial of degree not exceeding $m-1$ on each interval $(n, n+1)$. It is known [10] that if $\left(x_{n}\right)_{n=-\infty}^{\infty}$ is a bounded sequence, then there exists for each even $m>2$ a unique bounded cardinal spline $S_{m}$ which interpolates $\left(x_{n}\right)_{n=-\infty}^{\infty}$, i.e., such that $S_{m}(n)=x_{n}$ for all $n \in Z$.

TheOREM 3. Let $\nu \in M([0,2 \pi))$. Then for each even integer $m>2$, the bounded cardinal spline function of degree $m-1$ which interpolates the sequence $(\hat{\nu}(n))_{n=-\infty}^{\infty}$ is the Fourier transform of a measure $\omega_{m} \in M(R)$.

Proof. We apply the corollary with $G=R=\Gamma, H=2 \pi Z, H^{\perp}=Z$ and make the usual identification $R / 2 \pi Z=[0,2 \pi)$. Let $m$ be an even integer $>2$. Following [10] we set $M=\xi_{[-1 / 2,1 / 2]}$ and let $\hat{\delta}_{m}=M * M * \cdots * M$ ( $m$-fold convolution). Then $[10$, p. 408$],[9$, p. 175$] \hat{\delta}_{m} \in C_{00}(R)$ with supp $\hat{\delta}_{m} \subseteq[-m / 2, m / 2]$, and 
$\delta_{m}=([2 \sin (x / 2)] / x)^{m} \in L_{+}^{1}(R)$. Also $T \delta_{m}(x)=\Sigma_{n} \delta_{m}(x+2 \pi n)>0$ on $[0,2 \pi)$ $\left[9\right.$, Lemma 6]. Now, $\psi_{m}=\delta_{m} \cdot\left(\check{\beta}_{m} \circ \pi\right)$ and by (12) $T \psi_{m}=1$ on $[0,2 \pi)$. Thus,

$$
\hat{\psi}_{m}(n)=\left(T \psi_{m}\right)^{\wedge}(n)= \begin{cases}1 & \text { if } n=0, \\ 0 & \text { if } n \neq 0,\end{cases}
$$

and we see that $\hat{\psi}_{m}(x)=\Sigma_{n} \beta_{m}(n) \hat{\delta}_{m}(x-n)$ is precisely the function $L_{m}(x)$ of [10, (3.4), p. 414]. Hence, by [10, Theorem 3]

$$
\hat{\omega}_{m}(x)=\sum_{n} \hat{\nu}(n) \hat{\psi}_{m}(x-n)=\sum_{n} \hat{\nu}(n) L_{m}(x-n)
$$

is the unique bounded cardinal spline of degree $m-1$ which interpolates the sequence $(\hat{\nu}(n))_{n=-\infty}^{\infty}$.

ADDED IN PROOF. A general treatment of Theorem 1 in the case when $G / H$ is compact, due to L. Pigno and S. Saeki, has recently appeared. For an account of their version see C. C. Graham and O. C. McGehee, Essays in commutative harmonic analysis, Springer-Verlag, New York, 1979, p. 421.

\section{REFERENCES}

1. R. B. Burckel, Weakly almost periodic functions on semigroups, Gordon \& Breach, New York, 1970.

2. R. Doss, On the transform of a singular or an absolutely continuous measure, Proc. Amer. Math. Soc. 19 (1968), 361-363.

3. W. Feller, An introduction to probability theory and its applications, Vol. II, Wiley, New York, 1966.

4. R. R. Goldberg, Restrictions of Fourier transforms and extension of Fourier sequences, J. Approximation Theory 3 (1970), 149-155.

5. C. C. Graham and A. MacLean, A multiplier theorem for continuous measures, Studia Math. (to appear). 1968.

6. H. Reiter, Classical harmonic analysis and locally compact groups, Oxford Univ. Press, London,

7. W. Rudin, Fourier analysis on groups, Interscience, New York, 1962.

8. I. J. Schoenberg, Contributions to the problem of approximation of equidistant data by analytic functions: Part A, Quart. Appl. Math. 4 (1946), 45-99.

9. , Cardinal interpolation and spline functions, J. Approximation Theory 2 (1969), 167-206.

10. Cardinal interpolation and spline functions. II, Interpolation of data of power growth, J. Approximation Theory 6 (1972), 404-420.

11. Cardinal spline interpolation, Regional Conference Series in Applied Mathematics, No. 12 , Society for Industrial and Applied Mathematics (SIAM), Philadelphia, Pa., 1973.

Department of Mathematics, Wichita State University, Wichita, Kansas 67208 